Review

\title{
Real Time Dynamic Imaging and Current Targeted Therapies in the War on Cancer: A New Paradigm
}

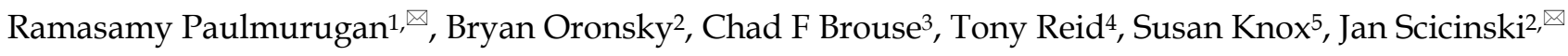 \\ 1. Department of Radiology, Stanford University School of Medicine, 1501 South California Avenue, Palo Alto, CA 94304. \\ 2. RadioRx, Inc, 800 W El Camino Real, Ste 180, Mountain View, CA 94040. \\ 3. University of Texas Southwestern Medical Center, 5323 Harry Hines Boulevard, Dallas, TX 75390. \\ 4. UCSD Moores Cancer Center, 3855 Health Sciences Drive, La Jolla, CA 92093. \\ 5. Department of Radiation Oncology, Stanford University Medical Center, 269 Campus Dr, CCSR South 1245, Stanford, CA 94305.
}

$\triangle$ Corresponding author: Ramasamy Paulmurugan, Ph.D. Department of Radiology, Stanford University School of Medicine, 1501, South California Avenue, \#2217, Palo Alto, CA 94304. Phone: 650-725-6097; Fax: 650-721-6921 Email: paulmur8@stanford.edu or Jan Scicinski, PhD, RadioRx, Inc, 800 W El Camino Real, Ste 180, Mountain View, CA 94040. jscicinski@radiorx.com.

(c) Ivyspring International Publisher. This is an open-access article distributed under the terms of the Creative Commons License (http://creativecommons.org/ licenses/by-nc-nd/3.0/). Reproduction is permitted for personal, noncommercial use, provided that the article is in whole, unmodified, and properly cited.

Received: 2012.12.05; Accepted: 2013.02.28; Published: 2013.05.25

\begin{abstract}
In biology, as every science student is made to learn, ontology recapitulates phylogeny. In medicine, however, oncology recapitulates polemology, the science of warfare: The medical establishment is transitioning from highly toxic poisons that kill rapidly dividing normal and malignant cells with little specificity to tailored therapies that target the tumors with the lethality of the therapeutic warhead. From the advent of the information age with the incorporation of high-tech intelligence, reconnaissance, and surveillance has resulted in "data fusion" where a wide range of information collected in near real-time can be used to redesign most of the treatment strategies currently used in the clinic. The medical community has begun to transition from the 'black box' of tumor therapy based solely on the clinical response to the 'glass box' of dynamic imaging designed to bring transparency to the clinical battlefield during treatment, thereby informing the therapeutic decision to 'retreat or repeat'. The tumor microenvironment is dynamic, constantly changing in response to therapeutic intervention, and therefore the therapeutic assessment must map to this variable and ever-changing landscape with dynamic and non-static imaging capabilities. The path to personalized medicine will require incorporation and integration of dynamic imaging at the bedside into clinical practice for real-time, interactive assessment of response to targeted therapies. The application of advanced real time imaging techniques along with current molecularly targeted anticancer therapies which alter cellular homeostasis and microenvironment can enhance therapeutic interventions in cancer patients and further improve the current status in clinical management of patients with advanced cancers.
\end{abstract}

Key words: Cancer, microRNA, cancer therapy, imaging, drug delivery.

\section{Introduction}

Real time dynamic imaging during cancer treatment cycles is crucial to understand and assess the real time therapeutic response of the tumors to a given cycle of therapy. Imaging 'snapshots' of the cancer before and after, rather than during, treatment cycles do not allow a continuously updated assess- ment and adjustment of treatment tactics for effective therapy. Currently, therapy is conducted with static imaging measurements of tumor volume before and after treatment with a, sometimes lengthy, 'information blackout' in between. This "conventional" state of information-gathering could be referred to as 
the stupidity of clinical intelligence. Traditional radiologic paradigms do not readily provide the means to inject new information into the collection plans after treatment is initiated.

According to the Response Evaluation Criteria in Solid Tumors (RECIST) and the World Health Organization (WHO) criteria, anchored in the mainstream of clinical trials, tumor response is determined anatomically with measurements taken at the start and the end of treatment. The tumor is imaged prematurely i.e. before the official end of treatment only if the physician suspects the patient has clinically progressed. Exceptions are few, but include Hodgkin 's disease where imaging is carried out to determine the total number of cycles of chemotherapy to be administered [1]. Also, in radiotherapy, intermediate imaging is carried out after a certain dose to determine the feasibility of shrinking the field [2]. However, in conventional treatment strategies, by the time the tumor is imaged after treatment, the war is over because adaptation mechanisms that lead to treatment resistance have often already evolved in the tumors. Multidrug resistance has been considered a major mechanism by which many cancers develop resistance to therapy. Tumors possess mixed population of normal and neoplastic cells. Even within the population of neoplastic cells in tumor there will be a wide variety of cells with various levels of responses to cancer therapy. When the tumor is treated with therapeutics, the sensitive cells respond and die while the recurrent tumor that appears from the resistant tumor cells tend to make the tumors drug resistant. To overcome this problem of drug resistance several pharmaceuticals were developed by various companies, which can sensitize the resistant cells in the first therapeutic regimen itself and kill both sensitive and resistant cells. In some other cases drugs have been developed to overcome the drug resistant mechanism and kill both sensitive and resistant cancer cells [3-5].

Tumors are characterized by an inherent adaptability to therapeutic perturbations. Appropriate treatment strategies that have traditionally been deployed based on best guesses and trial and error, therefore demand a power of adjustment that is at least equal to that of the tumor; the ability to stay one step ahead of the cancer resistance curve with clinical countermeasures requires the means and the flexibility to adjust and adapt the therapeutic modus operandi in real-time according to the response of the tumor. The transition from an era of "one size fits all" therapies to a model of personalized medicine requires a corresponding shift in radiological and imaging par- adigms from static to a real-time dynamic monitoring of response.

Dynamic and real-time image analysis is able to provide repeated estimates of metabolic parameters and microvascular function during therapy; in this way it is possible to take advantage of treatment-induced hemodynamic and metabolic changes as they occur to exploit the vulnerability of the tumor to other treatment modalities. In particular tumor blood flow changes can be used as an early indicator of effect to determine the optimal dose scheduling for combination therapies.

This review proposes a new paradigm for the integration of tumor imaging with cancer treatment. Combination therapeutic strategies with newer investigational compounds (e.g. Axitinib and RRx-001, in Phase III and Phase I trials, respectively, antioxidant chemo protective agents in different stages of clinical trials, and microRNAs in preclinical stage) are highlighted as examples for the incorporation of dynamic real-time monitoring into clinical studies to gauge anti-tumor response to therapy [6-12].

\section{Imaging Modalities}

Continuous assessment on a real-time basis with non-invasive imaging modalities such as positron emission tomography (PET) [13-15], magnetic resonance imaging (MRI) [16-18], and ultrasound (US) [19-21] provides a data composite of the continually evolving conditions and characteristics of the tumor in terms of vascularity, vascular permeability, blood flow, blood volume, hypoxia, metabolic activity, and cell turnover. However, data without analysis is meaningless. It is up to the clinician, working with the radiologist, to make sense of this raw jigsaw jumble of information, extrapolate a coherent clinical picture from disparate and fragmentary pieces of data and fill in the missing gaps with different modalities that complement and corroborate the heuristic assumptions. It is axiomatic that the tumor is dynamic, active and adaptive and therefore the clinical picture is continually evolving with the changes in therapeutic orientation and position. In this model of hide-and-seek the imaging assessments must be repeated iteratively and interactively to uncover the evasive response patterns of the tumor in order to rapidly react to them, allowing the imaging to drive the treatment (Figure 1). From global to local, each imaging modality provides a different and complementary field and level of view comparable to the space, air and ground reconnaissance platforms of the military. 


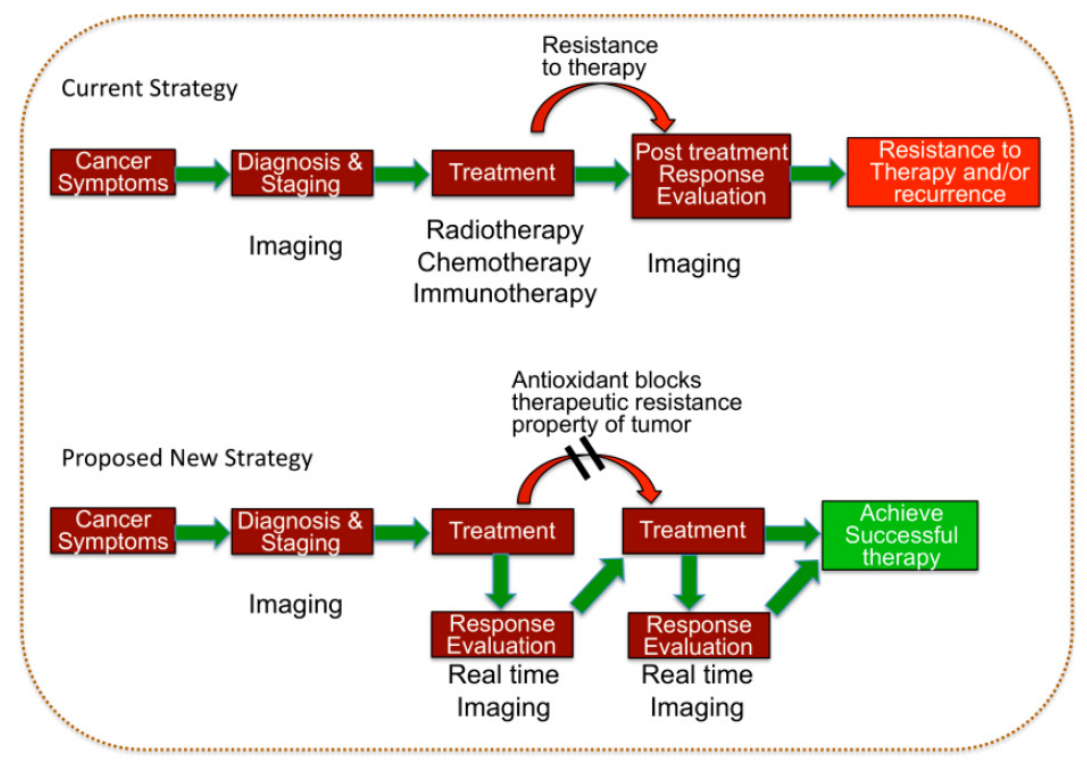

Figure I. Schematic illustration of the current therapeutic strategy used in the clinic and the proposed hypothetical model which can potentially improve the clinical outcome of various therapies for treating tumors of different sub-types.

\section{Positron Emission Tomography (PET) and Computed Tomography (CT)}

Like a satellite that looks down at the Earth's surface, providing a "bird's eye" view that is distinct from the "horizontal view" of the human eye, positron emission tomography (PET) and computed tomography (CT) provide a top-down perspective about anatomical and biological tumor information $[22,23]$.

PET is a nuclear medicine imaging technique that detects gamma rays emitted indirectly by a "tracer" or positron-emitting radionuclide [24, 25]. Depending on the tracer that is used, 3D images of a particular functional process of the tumor can be constructed. Currently there are a wide variety of tracer options that are specific to each type of measurement such as $\left[{ }^{18} \mathrm{~F}\right]$-fluoro-2-deoxy-D-glucose (FDG) for glucose metabolism [26-28], [ $\left.{ }^{15} \mathrm{O}\right]$-water for perfusion, $\left[\mathrm{C}^{15} \mathrm{O}\right]$-carbon monoxide for vascular volume, $\left[{ }^{18} \mathrm{~F}\right]-$ 3'-deoxy-3'-fluorothymidine (FLT) for tumor cell proliferation, and $\left[{ }^{18} \mathrm{~F}\right]-$ fluoromisonidazole (FMISO) for hypoxia [29]. The nitroimidazole, ${ }^{18} \mathrm{~F}-\mathrm{EF}-5$, is a new PET marker, currently under development for imaging hypoxia [30, 31] (Table 1). Following a scan, kinetic (compartment) models based on the principles of mass conservation are used to deduce physiological parameters from the time course of the local signal intensities (e.g. perfusion, blood volume) [32]. Compared to other imaging modalities, PET techniques that are extremely sensitive to the radiotracer, can provide excellent functional information but are limited in spatial resolution (3-4 $\mathrm{mm}$ ) [33].

Table I: PET tracer and target tumor parameters

\begin{tabular}{ll}
\hline PET tracer & Tumor parameter \\
\hline FDG: $\left[{ }^{18} \mathrm{~F}\right]$-fluoro-2-deoxy-D-glucose & Glucose metabolism \\
{$\left[{ }^{15} \mathrm{O}\right]$-water } & Perfusion \\
{$\left[\mathrm{C}^{15} \mathrm{O}\right]$-carbon monoxide } & Vascular volume \\
FLT: $\left[{ }^{18} \mathrm{~F}\right]-3^{\prime}$-deoxy-3'-fluorothymidine & $\begin{array}{l}\text { Tumor cell prolifera- } \\
\text { tion }\end{array}$ \\
FMISO: $\left[{ }^{18} \mathrm{~F}\right]$ - fluoromisonidazole & Hypoxia \\
F18 EF-5: [2-(2-nitro-1[H]-imidazol-1-yl)- & Hypoxia \\
N-(2,2,3,3,3-pentafluoropropyl)-acetamide] & \\
\hline
\end{tabular}

X-ray Computed tomography (CT) is an image reconstruction technique that assimilates projection-type data of x-ray photons that traversed the patient. When projection data is acquired from multiple spatial orientations around a single axis of rotation, a high resolution cross sectional image can be reconstructed. Digital geometry processing is used to generate a three-dimensional (3-D) image from a large series of two-dimensional (2-D) images or 'slices' [34]. CT has been widely used in various oncologic applications such as tumor diagnostic imaging, staging, and in decision-making process to evaluate tumors response to therapy. Current advances in $\mathrm{CT}$ technology such as the development of spiral-CT and dual energy CT has further advanced the use CT-imaging as a high speed technique with the potential to provide information regarding the tumor architecture 
before and after treatment as an indirect indication for measuring therapeutic response of tumor to a given therapy $[35,36]$.

PET/CT takes advantage of the sensitivity and functionality of PET imaging and the high spatial resolution of CT imaging [37]. CT is therefore commonly used in combination with FDG-PET and FLT-PET. PET and CT are combined into a single examination using an integrated PET/CT scanner that provides coregistered PET and CT images and, depending on the PET tracer, allows for the assessment of specific functional characteristics of the tumor with the contrast enhanced tomographic images from CT $[37,38]$. Recent advances in PET-CT imaging with the improved spatial resolution has made their use in the clinic for in room real time assessment of radiotherapy [39], one step closer to the use of a combination of real time imaging system with the treatment strategy in the assessment of tumor response to therapeutic interventions in cancer therapy, the eventual focus of this review.

\section{Magnetic Resonance Imaging (MRI)}

Similar to aerial reconnaissance, dynamic contrast enhanced Magnetic Resonance Imaging (MRI) provides a detailed anatomic and functional overview of particular targets such as tumors and this information can be correlated with the metabolic activity information provided by PET. MRI makes use of the property of nuclear magnetic resonance (NMR) to image nuclei of atoms and provides good contrast between the different soft tissues of the body making it especially useful in imaging tumors [40,41].

\section{Dynamic contrast enhanced MRI (DCE-MRI)}

Dynamic contrast-enhanced magnetic resonance imaging (DCE-MRI) relies on fast MRI sequences obtained before, during and after the bolus injection of the contrast agent. In this way, if conventional contrast-enhanced magnetic resonance imaging (MRI) is analogous to a snapshot, DCE-MRI is analogous to a movie, providing information about tumor vasculature as the contrast agent (CA) passes out of the blood vessels into the extracellular extravascular space [42, 43]. The most commonly used IV contrast agents are low molecular weight paramagnetic Gadolinium (Gd) compounds. By analyzing the uptake of the contrast agent into the tumor using an appropriate mathematical model, physiological parameters related to blood flow, vessel permeability or "leakiness", and tissue volume fractions of the extracellular extravascular leakage space (EES) can be extracted for each voxel or region of interest [44]. The parameters that are typically measured are: (1) the volume transfer constant $\left(K^{\text {trans }}\right)$ or $(2)$ the area under the initial contrast agent time curve (iAUC) [45]. $K^{\text {trans }}$ is an estimate of tumor vessel blood flow and permeability and iAUC reflects blood flow, vascular permeability, and the fraction of interstitial space [46]. The relationships between quantitative parameters (e.g. $K^{\text {trans }}$ ) and semi-quantitative parameters (e.g. iAUC) provide specific readouts of perfusion and permeability, which correlate with the tumor vascular physiology $[44,47]$. These parameters can be used for selecting optional therapeutic strategy needed for the treatment. DCE-MRI has been successfully used for evaluating invasive properties of breast cancer (ductal carcinoma in situ), prostate cancer and various other cancer subtypes. However the use of DCE-MRI has not been well explored for therapy monitoring, and in the evaluation of tumor response to various therapies.

\section{Ultrasound (US)}

\section{Contrast enhanced ultrasound (CEUS)}

In contrast to the bird's eye perspectives provided by PET and DCE-MRI, a more myopic view of tumor microvasculature, analogous to a narrowband acoustic ground-based military sensor, is provided by contrast enhanced ultrasound (CEUS). Due to its wide clinical availability, portability, non-invasiveness, ability to deliver multiple infusion of the contrast agent (CA), the absence of risk of nephrotoxicity, and lack of exposure to nuclear tracers or ionizing radiation, CEUS imaging represents one of the most promising tools to assess tumor hemodynamics [48, 49]. CEUS allows imaging of the tumor microvasculature by combining the use of specialized pulses of transmitted sound and processing of the received echoes with intravenously injected ultrasound contrast agents (USCA) that comprise of gas-containing thin-shelled microbubbles of approximately $1-5 \mu \mathrm{m}$ in diameter $[49,50]$. Their size allows the microbubbles to remain strictly intravascular where they can be detected with high sensitivity and specificity. The time-intensity curve in the region of interest following a bolus injection of USCA depends on tumor perfusion kinetics allowing a semi-quantitative measure of parameters such as peak intensity, time to peak (TTP) intensity, mean transit time (MTT), washout rate, and area under the total enhancement curve, with most scanners offering data analysis software. Another technique called destruction-reperfusion is more dependent on blood flow and fractional blood volume within the region of interest. Real-time observation of replenishment kinetics is acquired during USCA infusion to stabilize blood concentration, using low acoustic pressure (low MI- mechanical index) follow- 
ing a microbubble destruction pulse ("flash") [51].

The unique size of the microbubbles gives CEUS a major advantage over contrast-enhanced CT and DCE-MRI in characterizing tumors with increased vascular permeability and a large interstitial space (e.g. liver tumors). Whereas DCE-MRI may show prolonged enhancement due to contrast leakage into the tumor interstitium, CEUS demonstrates the washout phenomenon clearly and consistently [52]. CEUS imaging can be further enhanced by the use of specifically targeted microbubbles. For example microbubble targeted to the human kinase insert domain receptor (KDR, VEGFR-2), is under development for the specific imaging of tumor angiogenesis [53]. In some studies dual targeted microbubbles were developed to target two different proteins of tumor vasculature (VEGFR and alpha(v)beta(3) integrin) for imaging tumors with enhanced sensitivity [54]. CEUS also plays potential role in assessing tumor response to therapeutic interventions in cancer therapy. Since US-imaging system is widely available for clinical use, the optimization of this system for routine real time monitoring in cancer therapy in the clinic would be beneficial in determining the change in drug dose, number and frequency of administrations to achieve favorable outcome without allowing the tumor to develop resistance to a given therapy.

\section{US Elastography}

By applying pressure with the ultrasound transducer during real-time imaging, analogous to a physician's palpation, tissue displacement of one region relative to another is tracked in real time using ultrasonic correlation based methods to calculate the relative stiffness between these two regions as is done by most ultrasound based elastography devices, such as the General Electric LOGIQ E9 system. However, when applying rapid ultrasound pressure pulses of short durations to displace tissues, a shear wave is generated that is also detected by real time ultrasound. Acoustic Radiation Force Impulse Elastography (ARFI) provides specific information about tissue stiffness or strain of any region within the field of view and provides image contrast based on the shear wave velocity expressed in kiloPascals or $\mathrm{m} / \mathrm{sec}$ [55-57]. Several ultrasound scanners, such as the Aixplorer with SonicTouch (SuperSonic), and the Acuson S2000 with Virtual Touch (Siemens), implement ARFI technology for evaluation of deep tissues such as the liver parenchyma, kidneys, or tumors that are not accessible to superficial compression elastography techniques.

\section{The Use of Imaging Modalities in Com- bination with Therapeutic Strategies}

The war on cancer is fought on multiple fronts and in multiple patients but in most cases, like a multi-modal military operation, an armamentarium of therapeutic approaches is more successful than single-agent therapy. Timing is very important in combination therapy, where the optimal sequence of administration is determined by dynamic monitoring, which has the potential of in vivo synergy resulting in significantly improved patient outcomes. For example, angiogenesis inhibitors are rarely sufficiently active as monotherapy and are therefore typically combined with well-established modalities comprising mostly of cytotoxic agents [58,59]. The angiogenesis inhibitors, such as Avastin, are typically long acting, with prolonged pharmacodynamic half-lives designed to provide stable therapeutic levels of drug with infrequent dosing. This vascular-targeted strategy is based on the dual assumptions that sustained inhibition of angiogenesis is required for optimal activity and single mode angiogenesis inhibition is invulnerable to escape due to the redundancy of proangiogenic pathways.

\section{Combination therapeutic approach using Ax- itinib and FOLFOX}

In combination with chemotherapy, Reid and colleagues explored a novel therapeutic strategy with Axitinib, a small molecule tyrosine kinase inhibitor with a short half-life [60] that inhibits cellular signaling of multiple targets including VEGFR-1, VEGFR-2, VEGFR-3, platelet derived growth fact receptor (PDGFR), and cKIT (CD117) [61, 62]. In previous trials, axitinib dosing was designed to overcome the short half-life of the molecule and to maintain high and sustained levels of inhibition of VEGF and other targets thus maintaining a constant inhibition of blood flow into the tumor. However, with this dosing scenario, axitinib had failed to demonstrate activity either as a single agent in renal cell carcinoma or in combination with FOLFOX or FOLFIRI in advanced pancreatic cancer or metastatic colorectal cancer [63].

Using FLT-PET/CT, Reid and colleagues examined metabolic changes and demonstrated a decreased uptake of thymidine during the treatment phase (BID doses for 7 days) and increased uptake during the withdrawal phase (7 days). This FLT PET/CT data suggested a rebound hyperemia after the effects of Axitinib had dissipated. Based on the FLT-PET imaging for tumor proliferation and tumor blood flow measured by CT, FOLFOX was later administered during the putative tumor reperfusion 
phase. This new regimen resulted in markedly different outcomes compared to previous dosing modalities. The median progression-free survival (PFS) was nearly 6 months longer ( 9 versus 3 months) than expected for refractory colorectal cancer with this regimen. This prolonged median PFS was comparable to first-line therapy in previously untreated metastatic colorectal patients.

These data suggest that identifying tumor vasculature responses to vascular modifying agents on the fly is critical to optimally guide the timing of combination therapy with cytotoxic chemotherapeutics, to maximize antitumor activity through increased delivery of drugs after resumption of blood flow. Real time imaging with one or more of the above mentioned imaging modalities could help to identify optimal therapeutic intervention in this case and improve the outcome.

\section{Prospective combination therapeutic ap- proach using RRx-00 I}

$R R x-001$ is a novel intravenously administered pro-oxidant and nitric oxide (NO) modulating small molecule that is currently under evaluation in a Phase 1 study as a systemic anticancer agent [64]. From preclinical studies, the selectivity of RRx-001 is hypothesized to be based on the increased susceptibility of cancer cells to oxidative and nitrosative stress, possibly as a result of reduced expression of antioxidant defense enzymes such as superoxide dismutase-2, and the microvascular reactivity to nitric oxide modulation which can be exploited for selective manipulation of tumor blood flow. In addition, RRx-001 binds irreversibly to deoxyhemoglobin [65], modifying its nitrite reductase activity to endogenously generated high levels of NO under deeply hypoxic conditions [66] that are endemic to tumors.

The Phase 1 study of RRx-001 is designed to incorporate DCE-MRI and CEUS to study tumor blood flow kinetics and elastography, in addition to conventional tumor assessments, to assess the effects on the tumor vasculature. Changes in tumor vasculature, measured pre- and post-treatment will be used to design the optimal dosing schedule for Phase 2a radio- or chemotherapy combination studies. It is also under consideration for the implementation of real time imaging by CEUS to measure tumor blood flow kinetics and elastography before, during and after systemic administration of RRx-001 for continuously monitoring the evolving situation in the tumor to determine the therapy response criteria, and also to highlight the importance of real time imaging using CEUS for therapeutic monitoring in various other cancer therapies.

\section{Need of New Targets for Successful Therapy}

The major failure in treatment responses to anticancer drugs, either in monotherapy or as combination therapy, is due to resistance developed in cancer cells. This occurs by three major mechanisms: 1) activation of the multidrug resistant $\mathrm{p}$-glycoprotein efflux pump which rapidly clears chemotherapeutic drugs from the tumor, 2) cytochrome p450 mediated enzymatic neutralization of the effect of chemotherapeutics by phase I regulation, and 3) expression of phase II antioxidant enzymes which detoxify free radicals generated during therapy (Figure 2). Of these three mechanisms, the Phase II antioxidant detoxification mechanism is considered the major pathway responsible for effectively detoxifying the effect of cancer therapy, as this system is sensitive to and critical for maintaining cellular redox homeostasis, and a cascade of enzymes with different properties are rapidly activated to govern the effects from physical and chemical agents. Moreover, this particular mechanism is upregulated in cancer cells. Reactive oxygen species (ROS) are constantly generated in cells, including cancer cells. Cells maintain their redox homeostasis by developing mechanisms that protect them from oxidative stresses $[67,68]$. Since this mechanism is so well designed by nature to protect cells from free radicals produced during oxidative stresses in their aerobic environment, it also presents a major obstacle for tumor cell response to chemo- and radio- therapy. Chemotherapeutic drugs and radiotherapy normally induce ROS or release free radicals that cause cytotoxic damage and apoptosis in cancer cells. However, the therapy also activates the endogenous antioxidant detoxification mechanisms that hinder or counteract the therapeutic effects.

\section{Phase II antioxidant detoxification mechanism governed by Nuclear factor erythroid 2 related factor 2 (Nrf2) and resistance to cancer ther- apy}

Cells critically manage their intracellular ROS levels to maintain their redox homeostasis. Disruption of redox homeostasis by any means, especially in cancer cells after treating with anticancer drugs, can potentially activate this endogenous chemoprotective antioxidant mechanism. By activating this process, cells can develop a temporary adaptation to prevent the action of anticancer drugs. Nuclear factor erythroid 2 related factor 2 (Nrf2) is a transcription factor expressed in cells that protects cells from oxidative stresses by inducing antioxidant enzymes [69-71]. Oxidative stress increases when cells are ex- 
posed to any harsh conditions, such as when cancer cells encounter and react to anticancer drugs during treatment. Kelch-like ECH-associated protein 1 (Keap1) is important in rescuing cells from oxidative stresses [72-74]. Keap1 is an E3 ubiquitin ligase, which targets Nrf2 and constantly degrades its cytoplasmic form and maintains its nuclear form at a very low level to maintain cellular antioxidant enzymes at basal levels. However, when cells experience oxidative stress, Nrf2 gets phosphorylated by protein kinase C (PKC) [75] and trafficked into the nucleus, where it recruits its client protein (small-maf), binds to antioxidant response elements (ARE), and induces antioxidant enzyme expression. This basic mechanism of ROS detoxification is considerably up-regulated in cancers. It has recently been reported that different oncogenes (Kras, Braf, and myc) can induce Nrf2 transcription and promote ROS detoxification and tumorigenesis [76].

The use of chemo- and radio- therapies is obligatory for treating many cancers, including those not having specific targeted therapies, and advanced metastatic disease requiring palliative therapy. To improve the therapeutic efficiency, it is important for the drugs not to activate the tumor cells' antioxidant mechanism, which is generally up-regulated in tumor cells mainly due to their hypoxic microenvironments, and other up-regulated metabolic pathways [70, 76]. The Nrf2-Keap1-mediated antioxidant mechanism is a major inherent immune mechanism that protects cells from ROS and develops resistance in cancer therapy $[77,78]$. Developing drugs that can potentially bypass this process, or using a strategy that temporarily stops this endogenous process during treatment could result in more effective treatment options devised to achieve efficient therapy. Current drug developments and therapeutic strategies largely ignore the redox homeostasis aspects cells. Hence developing a combination therapy where the use of small molecule drugs that block the cellular phase II detoxification process during cancer therapy would enhance therapeutic outcome. Real time imaging with the potential to measure the level of activated endogenous antioxidant drug resistant mechanism while treating cancers with a combination therapy can be likely to be useful for adjusting the concentration and duration of antioxidants needed for treatment to improve therapeutic outcome.

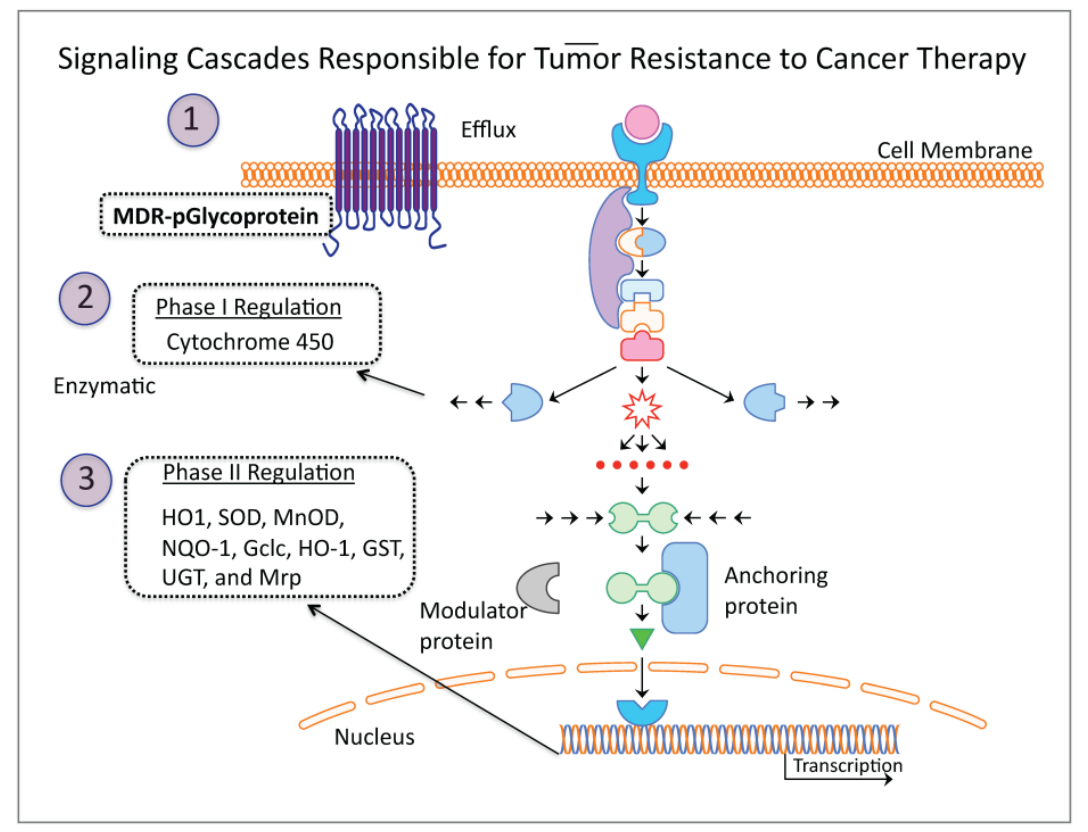

Figure 2. Schematic illustration of three major mechanisms contributes for tumor cells resistant to various therapeutic drugs and radiotherapy. By blocking these pathways temporarily during the treatments would potentially improve therapeutic outcome of various therapies used in the clinic for treating tumors of various sub-types.

\section{Emerging role of microRNAs in targeted cancer therapy}

Even though the use of antioxidants with current chemo- and radio- therapy can potentially improve the therapeutic efficiency in treating different cancers, blocking various endogenous drug resistant mechanisms before deploying therapy can even further improve the therapeutic response of tumors to a given therapy. MicroRNAs possess the ability to act as tu- 
mor suppressors by regulating the expression of oncogenes, apoptotic proteins, kinases, and other molecular mechanisms that can cause the onset of tumor development. MicroRNA let-7 was identified as a tumor suppressor in different types of tumors, such as breast cancer [79], colorectal cancer [80], and lung cancer [81]. MicroRNA-122, let-7 family, and microRNA-101 have been shown to function as tumor-suppressors in HCC [82]. Numerous studies support the idea that microRNAs act as tumor suppressors by regulating anti-apoptotic proteins [83-85]. MicroRNA-122, a liver-specific microRNA that is associated with the pathogenesis of HCC, and responsible for HCC resistance to conventional chemotherapy, was found to be downregulated in HCC. Overexpression of microRNA-122 in HCC cells sensitized them to adriamycin and vincristine by down regulating multidrug resistance (MDR)-related genes (MDR-1, GST-ח, MRP), anti-apoptotic gene (Bcl-w), and the cell cycle related gene (cyclin B1), thereby acting as a tumor suppressor [86].

Recent work by Hatziapostolou et al. has identified microRNA as a new player in HCC carcinogenesis, which can serve as a potential target for the treatment of HCC [87]. An inflammatory feedback loop circuit involving microRNA-24/microRNA629/HNF4alpha/microRNA-124/STAT3/IL-6R has been identified, which - upon activation - can suppress HNF4-alpha, thereby inducing HCC. Considering the therapeutic success and efficacy of microRNA-124, Hatziapostolou et al. further proposed its use in treatment of HCC. They found that supplementation of microRNA-124 directly reduced the expression of interleukin 6 receptor (IL6R), blocked the IL6-STAT3 mediated inflammatory loop, and reduced the tumor cell growth of HepG2 and SNU-449 human HCC xenografts in mice [87]. This result suggests the potential of using microRNA-124 therapy in HCC. Similarly, downregulation of tumor suppressor microRNAs such as microRNA-122, microRNA-101, and microRNA-203 has been associated with the development of HCC in patients. Over-expression of microRNA-221 and microRNA-21 has also been associated with tumor resistance to traditional chemotherapeutic drugs [88]. The finding that individual microRNAs target several hundred genes, regulate associated pathways involved in cellular pathogenesis and target microRNAs for the functional maintenance of cellular genes, further underscores the emerging importance of microRNA-mediated regulation in HCC $[89,90]$.

The up-regulation of microRNA-21 and microRNA-221 has been found to promote cell cycle progression, reduce apoptosis, and promote angio- genesis and invasion, while downregulation of tumor suppressor microRNAs such as microRNA-122, and microRNA-124 has been associated with the development of HCC in patients [91, 92]. Over-expression of microRNA-221 and microRNA-21 has also been associated with tumor cell resistance to traditional chemotherapeutic drugs [88]. Using a combined treatment approach, it is possible to target genes of these microRNAs (microRNA-21, microRNA-221, microRNA-122 and microRNA-124) simultaneously, and generate an additive effect, which can improve therapeutic outcome of HCC and other cancers when combined with different chemotherapeutic agents in combinations. The combined therapeutic approach will likely have a strong anti-proliferative effect on cancer cells, and will show substantial anti-metastatic and anti-invasive effects, in addition to making the cells sensitive to chemotherapeutic drugs. Although cellular microRNAs have been identified as potential targets for treating various cancers, the use of combination therapy where chemo- and radio- therapy combined with antioxidants, and real time imaging to measure evolving tumor response to therapy, has not even reached at its pre-clinical evaluation stage for their future use in successful clinical cancer therapies.

\section{Ultrasound-mediated drug delivery is a safe method for spatially localized drug admin- istration to achieve enhanced cancer therapy}

As stated above, the use of various combination therapies in conjunction with real time imaging can significantly improve cancer therapy. Unfortunately it doesn't address the issue of cancer therapy associated toxicity in non-target tissues. Normally toxicity to non-target tissues occurs only when higher doses of therapeutic agents are used for treatment. The uses of higher doses are essential to achieve efficient therapeutic index in tumors. Ultrasound (US) has been used for image-guided delivery of drugs [93-96]. Through a process termed "sonoporation," selective insonation of tissue actuates local formation of transient cell membrane microperforations, enhancing blood vessel wall permeability and facilitating the ingress of therapeutic agents into cells [97-100]. The putative primary mechanism for sonoporation is acoustic cavitation, whereby gas bodies oscillate and eventually collapse, releasing the energy necessary to induce transient cell membrane permeabilization. US-mediated drug delivery has shown to be markedly potentiated in the presence of microbubbles (MB), which serve as exogenous cavitation nuclei and reduce the US energy threshold for sonoporation to occur [97, 99-103]. From a clinically-translatable point of view, this approach is highly attractive because 
treatment 1) is highly-localized (only where US is applied) and guided by US imaging; 2) highly efficient, offering significantly higher local drug administration in the presence of US and MB; 3) shows sustained drug effect for several days or weeks following sonoporation [104-106]; and, 4) uses safe techniques (US does not expose patients to ionizing radiation; some MB formulations are already FDA approved, others are in Phase III clinical testing in the USA). However, this approach has not moved into the clinic yet and substantial improvements are needed to achieve sufficiently high treatment efficiency in vivo. One critical approach to improving the treatment efficiency is the optimization of treatment protocols, including acoustic parameters, microbubble selection, injection schemes, etc. A systematic parametric study tailored for tumor treatment is therefore critically needed. In addition, designing ultrasound transducers with the potential to deliver destruction pulses during drug delivery and CEUS-imaging potential to evaluate tumor response to therapy would be novel approach, which can significantly improve cancer therapy in the clinical.

\section{Conclusion}

In conclusion, the future of cancer therapy depends on the recognition of the tumor as a constantly moving target that evolves countermeasures from a diverse repertoire of evasive responses to survive. In this adaptive landscape, the therapeutic approach must co-evolve with the tumor, remaining sufficiently flexible to change or abandon the initial strategy as new circumstances or conditions arise. The need to mix and match different treatment options based on the cancer cell's response to therapy mandates a paradigm shift from traditional "static" imaging techniques to adaptive solutions that measure variations in parameters such as blood flow and volume and tissue stiffness in the tumor dynamically. Each of these methods non-invasively assesses different parameters that are slightly or wholly different, so the integration of dynamic functional imaging technologies into routine medical practice, as a powerful adjunct to the physical examination, has the potential to provide the clinician with a more complete and up-to-date perspective for the assessment of response to treatment and tumor behavior.

The example of Axitinib and RRx-001 demonstrate not only that real-time functional imaging is clinically feasible at both early and late stage trials, but also warranted as a personalized medicine blueprint for overcoming therapeutic resistance. More over the use of combination scheme where blocking the detoxification properties of cancer cells during therapy, and the use of microRNAs to change the cellular property of drug resistance and reducing metastatic and invasive properties of cancer cells can provide significant improvement in cancer therapy. In addition, the use of US-MB image guided drug delivery platform can enhance local delivery of custom combination of therapeutics (a combination of chemotherapeutic drugs, microRNAs and small molecule inhibitors of cellular phase II detoxification mechanisms) can, along with dynamic imaging that monitors therapeutic response, further change in the clinical management of this killer disease and save lives. Cancer may have fired the opening salvo but the clinician now has the ability to employ clinical countermeasures that are as adaptable, flexible and dynamic as the real-time imaging techniques available to assess the tumor's response.

\section{Competing Interests}

The authors have declared that no competing interest exists.

\section{References}

1. Ilivitzki A, Radan L, Ben-Arush M, Israel O, Ben-Barak A. Early interim FDG PET/CT prediction of treatment response and prognosis in pediatric Hodgkin disease-added value of low-dose CT. Pediatr Radiol 2013;43: 86-92.

2. Ding XP, et al. Feasibility of Shrinking Field Radiation Therapy through 18F-FDG PET/CT after 40 Gy for Stage III Non-Small Cell Lung Cancers. APJCP 2012;13: 319-323.

3. Bartsevich VV, Juliano RL. Regulation of the MDR1 gene by transcriptional repressors selected using peptide combinatorial libraries. Mol Pharmacol 2000;58: 1-10.

4. Marthinet E, Divita G, Bernaud J, Rigal D, Baggetto LG. Modulation of the typical multidrug resistance phenotype by targeting the MED-1 region of human MDR1 promoter. Gene Ther 2000;7: 1224-1233.

5. Tiberghien F, Kurome T, Takesako K, Didier A, Wenandy T, Loor F. Aureobasidins: structure-activity relationships for the inhibition of the human MDR1 P-glycoprotein ABC-transporter. J Med Chem 2000;43: 2547-2556.

6. Ladas EJ, Jacobson JS, Kennedy DD, Teel K, Fleischauer A, Kelly KM. Antioxidants and cancer therapy: a systematic review. J Clin Oncol 2004;22: 517-528.

7. Oronsky BT, Reid T, Knox SJ, Scicinski JJ. The scarlet letter of alkylation: a mini review of selective alkylating agents. Transl Oncol 2012;5: 226-229.

8. Ning S, Bednarski M, Oronsky B, Scicinski J, Saul G, Knox SJ. Dinitroazetidines are a novel class of anticancer agents and hypoxia-activated radiation sensitizers developed from highly energetic materials. Cancer Res 2012;72: 2600-2608.

9. Scicinski J, et al. Preclinical evaluation of the metabolism and disposition of RRx-001, a novel investigative anticancer agent. Drug Metab Dispos 2012;40: 1810-1816.

10. Taneja SS. Re: Comparative effectiveness of axitinib versus sorafenib in advanced renal cell carcinoma (AXIS): a randomised phase 3 trial. J Urol 2012;188: 412-413.

11. Kenney PA, Wood CG. Words of wisdom. Re: Comparative effectiveness of axitinib versus sorafenib in advanced renal cell carcinoma (AXIS): a randomised phase 3 trial. Eur Urol 2012;62: 182-183.

12. Rini BI, et al. Comparative effectiveness of axitinib versus sorafenib in advanced renal cell carcinoma (AXIS): a randomised phase 3 trial. Lancet 2011;378: 1931-1939.

13. Solanki AA, et al. The utility of FDG-PET for assessing outcomes in oligometastatic cancer patients treated with stereotactic body radiotherapy: a cohort study. Radiat Oncol 2012;7: 216

14. Matsumoto I, et al. Usefulness of fluorine-18 fluorodeoxyglucose-positron emission tomography in management strategy for thymic epithelial tumors. Ann Thorac Surg 2013;95: 305-310. 
15. Li X, et al. An algorithm for longitudinal registration of PET/CT images acquired during neoadjuvant chemotherapy in breast cancer: preliminary results. EJNMMI Res 2012;2: 62.

16. Kim HS, Kim CK, Park BK, Huh SJ, Kim B. Evaluation of therapeutic response to concurrent chemoradiotherapy in patients with cervical cancer using diffusion-weighted MR imaging. J Magn Reson Imaging 2013;37: 187-193.

17. Saito N, et al. Posttreatment CT and MR imaging in head and neck cancer: what the radiologist needs to know. Radiographics 2012;32: 1261-1282.

18. Hylton NM, et al. Locally advanced breast cancer: MR imaging for prediction of response to neoadjuvant chemotherapy--results from ACRIN 6657/I-SPY TRIAL. Radiology 2012;263: 663-672.

19. Mercier L, Del Maestro RF, Petrecca K, Araujo D, Haegelen C, Collins DL. Online database of clinical MR and ultrasound images of brain tumors. Med Phys 2012;39: 3253-3261.

20. Smyslenova MV, Tarasenko SV, Shipkova TP, Rudnev AI, Faskhutdinov DK. [Capabilities of ultrasound study in the differential diagnosis of neoplasms of the major salivary glands]. Vestn Rentgenol Radiol 2012;: 8-11.

21. Yang X, et al. Ultrasound GLCM texture analysis of radiation-induced parotid-gland injury in head-and-neck cancer radiotherapy: an in vivo study of late toxicity. Med Phys 2012;39: 5732-5739.

22. Pauwels EK, Coumou AW, Kostkiewicz M, Kairemo K. [F]Fluoro-2-Deoxy-D-Glucose Positron Emission Tomography/Computed Tomography Imaging in Oncology: Initial Staging and Evaluation of Cancer Therapy. Med Princ Pract. 2013

23. Abramyuk A, Appold S, Zophel K, Baumann M, Abolmaali N. Modification of staging and treatment of head and neck cancer by FDG-PET/CT prior to radiotherapy. Strahlenther Onkol 2013.

24. Lodge MA, Badawi RD, Gilbert R, Dibos PE, Line BR. Comparison of 2-dimensional and 3-dimensional acquisition for 18F-FDG PET oncology studies performed on an LSO-based scanner. J Nucl Med 2006;47: 23-31.

25. Hawkins RA, Hoh CK. PET FDG studies in oncology. Nucl Med Biol 1994;21: 739-747.

26. Sauter AW, et al. Correlation between [(18)F]FDG PET/CT and volume perfusion CT in primary tumours and mediastinal lymph nodes of non-small-cell lung cancer. Eur J Nucl Med Mol Imaging. 2013.

27. Malle P, Sorschag M, Gallowitsch HJ. FDG PET and FDG PET/CT in patients with gastrointestinal stromal tumours. Wien Med Wochenschr 2012;162: 423-429.

28. Bertagna F, Bosio G, Orlando E, Biasiotto G, Giubbini R. Role of F-18-FDG-PET/CT in restaging of patients affected by gastrointestinal stromal tumours (GIST). Nucl Med Rev Cent East Eur 2010;13: 76-80.

29. Asselin MC, O'Connor JP, Boellaard R, Thacker NA, Jackson A. Quantifying heterogeneity in human tumours using MRI and PET. Eur J Cancer 2012;48: 447-455.

30. Evans SM, et al. EF5 binding and clinical outcome in human soft tissue sarcomas. Int J Radiat Oncol Biol Phys 2006;64: 922-927.

31. Ziemer LS, et al. Noninvasive imaging of tumor hypoxia in rats using the 2-nitroimidazole 18F-EF5. Eur J Nucl Med Mol Imag 2003;30: 259-266.

32. Laking GR, West C, Buckley DL, Matthews J, Price PM. Imaging vascular physiology to monitor cancer treatment. Crit Rev Oncol Hematol 2006;58: 95-113.

33. Langen $\mathrm{KJ}$, et al. 3-[123I]iodo-alpha-methyltyrosine and [methyl-11C]-L-methionine uptake in cerebral gliomas: a comparative study using SPECT and PET. J Nucl Med 1997;38: 517-522.

34. Ter-Pogossian MM. Basic principles of computed axial tomography. Semin Nucl Med 1977;7: 109-127.

35. De Cecco CN, et al. Dual-energy CT: oncologic applications. AJR Am J Roentgenol 2012;199: S98-S105.

36. Kadowaki K, et al. Helical CT imaging of gastric cancer: normal wall appearance and the potential for staging. Radiat Med 2000;18: 47-54.

37. Kim EE. Whole-body positron emission tomography and positron emission tomography/computed tomography in gynecologic oncology. Int J Gynecol Cancer 2004;14: 12-22.

38. Bowen SR, et al. Challenges and opportunities in patient-specific, motion-managed and PET/CT-guided radiation therapy of lung cancer: review and perspective. Clin Transl Med 2012;1: 18

39. Min CH, et al. Clinical Application of In-Room Positron Emission Tomography for In Vivo Treatment Monitoring in Proton Radiation Therapy. Int J Radiat Oncol Biol Phys. 2013.

40. Paushter DM, Modic MT, Borkowski GP, Weinstein MA, Zeman RK. Magnetic resonance. Principles and applications. Med Clin North Am 1984;68: 1393-1421.

41. Chilton HM, Ekstrand KE. Principles and applications of nuclear magnetic resonance imaging. Am J Hosp Pharm 1984;41: 763-768.
42. Moon M, Cornfeld D, Weinreb J. Dynamic contrast-enhanced breast MR imaging. Magn Reson Imaging Clin N Am 2009;17: 351-362.

43. Morakkabati-Spitz N, Schild HH, Leutner CC, von Falkenhausen M, Lutterbey G, Kuhl CK. Dynamic contrast-enhanced breast MR imaging in men: preliminary results. Radiology 2006;238: 438-445.

44. Tunariu N, Kaye SB, Desouza NM. Functional imaging: what evidence is there for its utility in clinical trials of targeted therapies? Br J Cancer 2012;106: 619-628.

45. Leach MO, et al. Assessment of antiangiogenic and antivascular therapeutics using MRI: recommendations for appropriate methodology for clinical trials. Br J Radiol 76 Spec No 2003;1: S87-91.

46. Yankeelov TE, Gore JC. Dynamic Contrast Enhanced Magnetic Resonance Imaging in Oncology: Theory, Data Acquisition, Analysis, and Examples. Curr Med Imaging Rev 2009;3: 91-107.

47. Rehman S, Jayson GC. Molecular imaging of antiangiogenic agents. Oncologist 2005;10: 92-103.

48. Quaia E. Assessment of tissue perfusion by contrast-enhanced ultrasound. Eur Radiol 2010;21: 604-615.

49. Delorme S, Krix M. Contrast-enhanced ultrasound for examining tumor biology. Cancer Imaging 2006;6: 148-152.

50. Wilson SR, Burns PN. Microbubble-enhanced US in body imaging: what role? Radiology 2010;257: 24-39.

51. Krix M, et al. Quantification of perfusion of liver tissue and metastases using a multivessel model for replenishment kinetics of ultrasound contrast agents. Ultrasound Med Biol 2004;30: 1355-1363.

52. Jang HJ, Yu H, Kim TK. Contrast-enhanced ultrasound in the detection and characterization of liver tumors. Cancer Imaging 2009;9: 96-103.

53. Pysz MA, Foygel K, Rosenberg J, Gambhir SS, Schneider M, Willmann JK. Antiangiogenic cancer therapy: monitoring with molecular US and a clinically translatable contrast agent (BR55). Radiology 2010;256: 519-527.

54. Willmann JK, et al. Dual-targeted contrast agent for US assessment of tumor angiogenesis in vivo. Radiology 2008;248: 936-944.

55. Sporea I, Sirli R, Popescu A, Danila M. Acoustic Radiation Force Impulse (ARFI)--a new modality for the evaluation of liver fibrosis. Med Ultrason 2010;12: 26-31.

56. Friedrich-Rust $\mathrm{M}$, et al. Liver fibrosis in viral hepatitis: noninvasive assessment with acoustic radiation force impulse imaging versus transient elastography. Radiology 2009;252: 595-604.

57. Nightingale K, Soo MS, Nightingale R, Trahey G. Acoustic radiation force impulse imaging: in vivo demonstration of clinical feasibility. Ultrasound Med Biol 2002;28: 227-235.

58. Beretta GD, et al. FOLFIRI + bevacizumab as second-line therapy for metastatic colorectal cancer pretreated with oxaliplatin: a pooled analysis of published trials. Med Oncol 2013;30: 486.

59. Sanchez-Rovira $P$, et al. Bevacizumab plus preoperative chemotherapy in operable HER2 negative breast cancer: biomarkers and pathologic response. Clin Transl Oncol. 2013.

60. Patson B, R BC, Olszanski AJ. Pharmacokinetic evaluation of axitinib. EXPERT OPIN DRUG MET 2012;8: 259-270.

61. Sharma S, et al. A phase I study of axitinib (AG-013736) in combination with bevacizumab plus chemotherapy or chemotherapy alone in patients with metastatic colorectal cancer and other solid tumors. Ann Oncol 2009;21: 297-304.

62. Hoh C, et al. Axitinib inhibition of $[18 \mathrm{~F}]$ fluorothymidine (FLT) uptake in patients (pts) with colorectal cancer (CRC): Implications for cytotoxic chemotherapy combinations. J Clin Oncol 2011;29: abstr359.

63. Bendell J, et al. Axitinib or bevacizumab (bev) plus FOLFOX or FOLFIRI as second-line therapy in patients (pts) with metastatic colorectal cancer (mCRC). J Clin Oncol 2011;29: abstr478.

64. Ning S, Bednarski M, Oronsky B, Scicinski J, Saul G, Knox S. Dintroazetidines are a Novel Class of Anticancer Agents and Hypoxia-activated Radiation Sensitizers Developed from Highly Energetic Materials. Cancer Res 2012;72: 2600-2608.

65. Scicinski J, et al. Disposition of 14C-RRx-001 in Rats after A Single Intravenous Administration and in Blood from Rats, Dogs, Monkeys, and Humans. In 17th North American Regional ISSX Meeting, P81, Atlanta, GA. 2011.

66. Fens MHAM, et al. NO or No NO, Increased Reduction of Nitrite to Nitric Oxide by Modified Red Blood Cells. Blood (ASH Annual Meeting Abstracts) 2011;118: 2125.

67. Foyer $\mathrm{CH}$, Noctor $\mathrm{G}$. Redox homeostasis and antioxidant signaling: a metabolic interface between stress perception and physiological responses. Plant Cell 2005;17: 1866-1875.

68. Cullinan SB, Diehl JA. PERK-dependent activation of Nrf2 contributes to redox homeostasis and cell survival following endoplasmic reticulum stress. J Biol Chem 2004;279: 20108-20117. 
69. Li Y, Jaiswal AK. Human antioxidant-response-element-mediated regulation of type $1 \mathrm{NAD}(\mathrm{P}) \mathrm{H}$ :quinone oxidoreductase gene expression. Effect of sulfhydryl modifying agents. Eur J Biochem 1994;226: 31-39.

70. Venugopal R, Jaiswal AK. Nrf1 and Nrf2 positively and c-Fos and Fra1 negatively regulate the human antioxidant response element-mediated expression of NAD $(\mathrm{P}) \mathrm{H}$ :quinone oxidoreductase1 gene. Proc Natl Acad Sci U S A 1996;93: 14960-14965.

71. Nioi P, Nguyen T, Sherratt PJ, Pickett CB. The carboxy-terminal Neh3 domain of Nrf2 is required for transcriptional activation. Mol Cell Biol 2005;25: 10895-10906

72. Moi P, Chan K, Asunis I, Cao A, Kan YW. Isolation of NF-E2-related factor 2 (Nrf2), a NF-E2-like basic leucine zipper transcriptional activator that binds to the tandem NF-E2/AP1 repeat of the beta-globin locus control region. Proc Natl Acad Sci U S A 1994;91: 9926-9930.

73. Yamamoto T, et al. Physiological significance of reactive cysteine residues of Keap1 in determining Nrf2 activity. Mol Cell Biol 2008;28: 2758-2770.

74. Sekhar KR, Rachakonda G, Freeman ML. Cysteine-based regulation of the CUL3 adaptor protein Keap1. Toxicol Appl Pharmacol 2010;244: 21-26.

75. Bloom DA, Jaiswal AK. Phosphorylation of Nrf2 at Ser40 by protein kinase $\mathrm{C}$ in response to antioxidants leads to the release of Nrf2 from INrf2, but is not required for Nrf2 stabilization/accumulation in the nucleus and transcriptional activation of antioxidant response element-mediated NAD(P)H:quinone oxidoreductase-1 gene expression. J Biol Chem 2003;278: 44675-44682.

76. DeNicola GM, et al. Oncogene-induced Nrf2 transcription promotes ROS detoxification and tumorigenesis. Nature 2011;475: 106-109.

77. Kong Q, Lillehei KO. Antioxidant inhibitors for cancer therapy. Med Hypotheses 1998;51: 405-409.

78. Sharifi N. Commentary: Antioxidants for cancer: new tricks for an old dog? Oncologist 2009;14: 213-215.

79. $\mathrm{Yu} \mathrm{F}$, et al. let-7 regulates self renewal and tumorigenicity of breast cancer cells. Cell 2007;131: 1109-1123.

80. Akao Y, Nakagawa Y, Naoe T. let-7 microRNA functions as a potential growth suppressor in human colon cancer cells. Biol Pharm Bull 2006;29: 903-906.

81. Johnson SM, et al. RAS is regulated by the let-7 microRNA family. Cell 2005;120: 635-647.

82. Saito $Y$, et al. MicroRNAs in Hepatobiliary and Pancreatic Cancers. Front Genet 2011;2: 66 .

83. Jiang S, et al. MicroRNA-155 functions as an OncomiR in breast cancer by targeting the suppressor of cytokine signaling 1 gene. Cancer Res 2010;70: 3119-3127.

84. Jing Z, Nan KJ, Hu ML. Cell proliferation, apoptosis and the related regulators p27, p53 expression in hepatocellular carcinoma. World J Gastroenterol 2005;11: 1910-1916.

85. Wang $\mathrm{XF}$, et al. MiR-181d acts as a tumor suppressor in glioma by targeting K-ras and Bcl-2. J Cancer Res Clin Oncol 2012;138: 573-584.

86. Xu Y, et al. MicroRNA-122 sensitizes HCC cancer cells to adriamycin and vincristine through modulating expression of MDR and inducing cell cycle arrest. Cancer Lett 2011;310: 160-169.

87. Hatziapostolou M, et al. An HNF4alpha-miRNA inflammatory feedback circuit regulates hepatocellular oncogenesis. Cell 2011;147: 1233-1247.

88. Kutanzi KR, Yurchenko OV, Beland FA, Checkhun VF, Pogribny IP. MicroRNA-mediated drug resistance in breast cancer. Clin Epigenetics 2011;2: 171-185

89. Bushati N, Cohen SM. microRNA functions. Annu Rev Cell Dev Biol 2007;23: 175-205.

90. Lewis BP, Burge CB, Bartel DP. Conserved seed pairing, often flanked by adenosines, indicates that thousands of human genes are microRNA targets. Cell 2005;120: 15-20.

91. Stenvang J, Petri A, Lindow M, Obad S, Kauppinen S. Inhibition of microRNA function by antimiR oligonucleotides. Silence 2012;3: 1.

92. Zhang G, Wang Q, Xu R. Therapeutics Based on microRNA: A New Approach for Liver Cancer. Curr Genomics 2010;11: 311-325.

93. Deckers R, Moonen CT. Ultrasound triggered, image guided, local drug delivery. J Control Release 2010;148: 25-33.

94. Phillips LC, Dhanaliwala AH, Klibanov AL, Hossack JA, Wamhoff BR. Focused ultrasound-mediated drug delivery from microbubbles reduces drug dose necessary for therapeutic effect on neointima formation--brief report. Arterioscler Thromb Vasc Biol 2011;31: 2853-2855.

95. Phillips LC, Klibanov AL, Wamhoff BR, Hossack JA. Localized ultrasound enhances delivery of rapamycin from microbubbles to prevent smooth muscle proliferation. J Control Release 2011;154: 42-49.

96. Wang DS, et al. Enhanced Ultrasound-Mediated Gene Delivery Using Cationic Microbubbles. Radiology; in press.
97. Miller DL, Pislaru SV, Greenleaf JE. Sonoporation: mechanical DNA delivery by ultrasonic cavitation. Somat Cell Mol Genet 2002;27: 115-134.

98. Tachibana K, Uchida T, Ogawa K, Yamashita N, Tamura K. Induction of cell-membrane porosity by ultrasound. Lancet 1999;353: 1409.

99. Brayman AA, Coppage ML, Vaidya S, Miller MW. Transient poration and cell surface receptor removal from human lymphocytes in vitro by 1 MHz ultrasound. Ultrasound Med Biol 1999;25: 999-1008.

100. Duvshani-Eshet M, Baruch L, Kesselman E, Shimoni E, Machluf M. Therapeutic ultrasound-mediated DNA to cell and nucleus: bioeffects revealed by confocal and atomic force microscopy. Gene Ther 2006;13: 163-172.

101. Kimmel E. Cavitation bioeffects. Crit Rev Biomed Eng 2006;34: 105-161.

102. Schlicher RK, Radhakrishna H, Tolentino TP, Apkarian RP, Zarnitsyn V, Prausnitz MR. Mechanism of intracellular delivery by acoustic cavitation. Ultrasound Med Biol 2006;32: 915-924.

103. Greenleaf WJ, Bolander ME, Sarkar G, Goldring MB, Greenleaf JF. Artificial cavitation nuclei significantly enhance acoustically induced cell transfection. Ultrasound Med Biol 1998;24: 587-595.

104. Chumakova OV, et al. Composition of PLGA and PEI/DNA nanoparticles improves ultrasound-mediated gene delivery in solid tumors in vivo. Cancer Lett 2008;261: 215-225.

105. Figueiredo M, Esenaliev R. PLGA Nanoparticles for Ultrasound-Mediated Gene Delivery to Solid Tumors. J Drug Deliv 2012;2012: 767839.

106. Paulmurugan R, Sekar NM, Sekar TV. Biodegradable polymer nanocarriers for therapeutic antisense microRNA delivery in living animals. Proceedings of SPIE 8232. 2012. 